Acta Crystallographica Section D

Biological Crystallography

ISSN 1399-0047

aPhysical Biosciences Division, Lawrence Berkeley National Laboratory, Berkeley, CA 94720-8235, USA, 'bepartment of Haematology, University of Cambridge, Cambridge Institute for Medical Research, Wellcome Trust/MRC Building, Cambridge CB2 OXY, England, 'Los Alamos National Laboratory, Los Alamos, NM 87545-0001, USA, and department of Bioengineering, University of California at Berkeley, Berkeley, CA 94720-1762, USA

Correspondence e-mail: nechols@lbl.gov, nwmoriarty@lbl.gov, pdadams@lbl.gov

\title{
Automating crystallographic structure solution and refinement of protein-ligand complexes
}

High-throughput drug-discovery and mechanistic studies often require the determination of multiple related crystal structures that only differ in the bound ligands, point mutations in the protein sequence and minor conformational changes. If performed manually, solution and refinement requires extensive repetition of the same tasks for each structure. To accelerate this process and minimize manual effort, a pipeline encompassing all stages of ligand building and refinement, starting from integrated and scaled diffraction intensities, has been implemented in Phenix. The resulting system is able to successfully solve and refine large collections of structures in parallel without extensive user intervention prior to the final stages of model completion and validation.

\section{Introduction}

One of the most important developments in macromolecular crystallography over the past 15 years has been the development of increasingly automated computational tools that are significantly more rigorous and self-diagnostic, thereby decreasing the manual effort involved in structure solution. Besides general improvements in the capabilities of individual components of structure-determination pipelines, especially in the area of automated building (Perrakis et al., 1999; Cowtan, 2006; DiMaio et al., 2006; Terwilliger et al., 2008), a number of sophisticated pipelines encompassing multiple steps have been described (Bricogne et al., 2003; Holton \& Alber, 2004; Ness et al., 2004; Panjikar et al., 2005; Keegan \& Winn, 2007; Terwilliger et al., 2009). These projects are especially focused on accelerating the early stages of the process (starting either from raw diffraction images or reduced data), with the goal of obtaining an unambiguous partial model with minimal user intervention. Completing the structure is left to the crystallographer and still remains a largely manual procedure.

In conjunction with these software projects, the productivity of synchrotron beamlines has progressed, driven by a combination of brighter radiation sources (Carwardine et al., 2003), improved detector hardware (Broennimann et al., 2006), and automated sample mounting and data collection (Karain et al., 2002; Cipriani et al., 2006; Ueno et al., 2006; Grochulski et al., 2012). These developments are particularly useful for structure-based drug discovery, which has prompted pharmaceutical companies to build beamlines dedicated to this purpose. Although these structures encompass a relatively small number of target proteins deemed to be of therapeutic utility, the throughput from industrial projects has been estimated at upwards of 10000 structures per year (Wasserman et
Received 10 September 2013 Accepted 7 October 2013 
al., 2012). With nearly $600 \mathrm{X}$-ray crystal structures deposited in the wwPDB (Berman et al., 2003), the impact of crystallographic studies on the discovery and characterization of the numerous FDA-approved HIV-1 protease inhibitors is undeniable (Wlodawer, 2002). Similar outcomes with other diseases [e.g. hepatitis C virus (Kanda et al., 2013) and chronic myelogenous leukemia (Milojkovic \& Apperley, 2008)] are on the way to being realised.

An essential task in structure-based drug discovery is the placement of functionally relevant ligands into residual electron density. This has been aided in recent years by a large number of software packages, including $X$-LIGAND (Oldfield, 2001), ARP/wARP (Zwart et al., 2004), LigandFit (Terwilliger et al., 2006), AFITT (Wlodek et al., 2006), RhoFit (Global Phasing Ltd) and PrimeX (Bell, Cao et al., 2012). Most ligand-fitting programs incorporate a local real-space refinement step after the initial placement. To varying degrees, most also integrate with ligand-parameterization, model-building and refinement software. However, the very repetitive workflow of high-throughput co-crystallography generally remains a series of discrete steps. Although pharmaceutical companies often develop proprietary internal pipelines (Kroemer et al.,

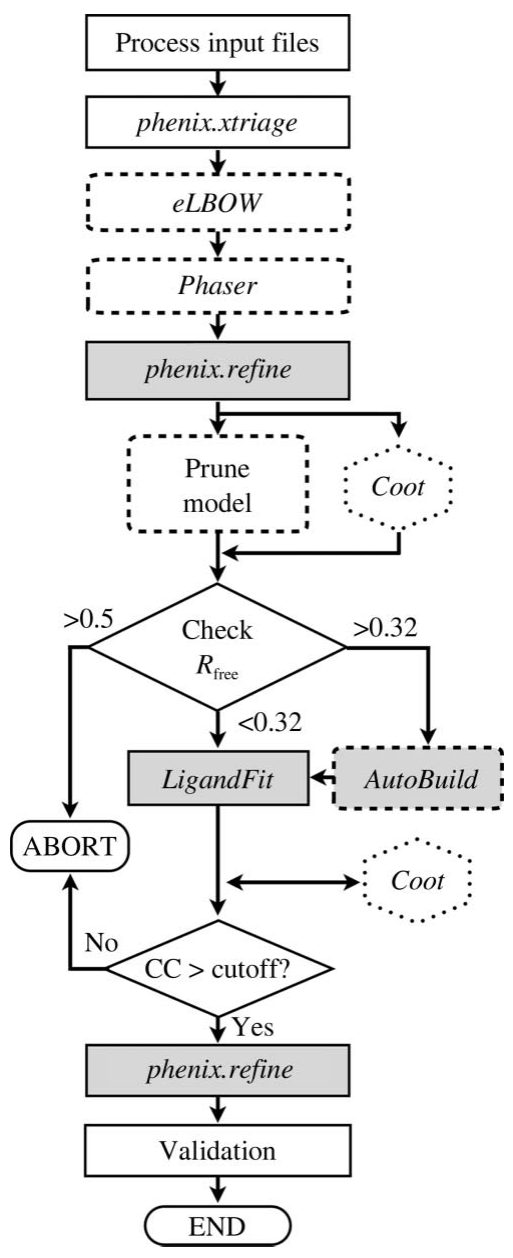

Figure 1

A schematic of the pipeline workflow. Optional modules are highlighted with dashed borders and multiprocessor-aware modules are designated by gray shading. The Coot steps are only invoked in interactive mode.
2004; Mooij et al., 2006; Davies \& Tickle, 2012; Wasserman et al., 2012), and several independent groups have automated parts of the process (e.g. Tsai et al., 2013; Sharff et al., 2012), most of these systems are either integrated with beamline automation or are not readily available to the broad crystallographic community.

While many who have been tasked with solving a large number of crystal structures have developed some means to solve the $n$th structure faster and more easily than the previous $n-1$ structures out of sheer necessity, the task of developing a truly generic and robust pipeline is more difficult than might at first be imagined. It can be relatively straightforward to optimize a pipeline for one class of structures; however, to make it sufficiently robust to handle very different classes of structures and different qualities of crystallographic data is non-trivial. Consistent with the Pareto principle (Juran \& Gryna, 1988), or the 80-20 rule, much of the development effort remains dedicated to making a small number of cases work. This disparity between effort and percentage success can be explained by the observation that in the course of a structure determination, the crystallographer must make numerous decisions. Many of these decisions rely on his or her experience and are difficult to codify, especially when a program is restricted to only the current coordinate and diffraction data. Even crystallographic steps that are often taken for granted (e.g. space-group determination and molecular replacement) are difficult to automate universally because many parameters (e.g. solvent content) are only guidelines and because of the pervasive extent to which prior knowledge is naturally and unconsciously utilized.

Here, we describe an integrated pipeline for protein-ligand structure determination as part of the Phenix suite (Adams et al., 2010) that was written to specifically target these historically difficult steps. This pipeline was constructed based on several previously described programs that were intentionally designed around a common framework with future automation in mind (Terwilliger et al., 2006; McCoy et al., 2007; Moriarty et al., 2009; Afonine et al., 2012). Considerable effort was made to codify the decision-making steps used by experienced crystallographers as they weighed intermediate results against the relevant guideline(s). Starting from processed data, a closely related molecular-replacement search model and basic chemical information about the target ligand, the program is capable of producing high-quality and nearly complete structures with minimal user intervention in many cases. Integrated validation tools (Chen et al., 2010) assist the user with quality control and completion of the resulting structures. The pipeline was benchmarked against several collections of structure-based drug-discovery proteinligand complexes and a representative sample of the Iridium database of curated protein-ligand structures (Warren et al., 2012). In order to ensure no advantage from hindsight, the pipeline was given the same search model and structure factors as used for the published structure determination. The pipeline is able to solve many ligand-bound structures and in some cases can produce results that rival, if not exceed, those of the original deposition. 


\section{Methods}

\subsection{Basic design}

The software (phenix.ligand_pipeline) is implemented in Python and is designed to be entirely self-contained within the Phenix suite with no external dependencies. An overview of the steps taken is shown in Fig. 1. The individual steps were encapsulated in modular code so they could be used iteratively and in different workflows. The approach could be extended in the future to adopt a more general-purpose automation framework where components could be removed or added (Tsai et al., 2013).

In most cases, the program runs with minimal configuration. The only mandatory inputs are processed data (scaled intensities or amplitudes, in any commonly used format), a starting model for molecular replacement (or, if isomorphous, molecular substitution) and a source of ligand geometry information such as a SMILES string or file (Weininger, 1988), a MOL2 or restraints CIF file, or a PDB residue code that directs $e L B O W$ (Moriarty et al., 2009) to use the entry in the Chemical Components Dictionary (Henrick et al., 2008). If a directory path is given as input, the program attempts to automatically determine the appropriate input files by scanning the directory contents. Although the processing of raw diffraction images is currently outside the scope of Phenix, the program could be extended to run in conjunction with existing automated data-processing pipelines (Winter, 2010; Vonrhein et al., 2011).

2.1.1. Data setup and analysis. An initial step converts the diffraction data to amplitudes in MTZ format as necessary. $R_{\text {free }}$ flags are either imported or generated if absent. Following this conversion, the data quality is assessed using phenix.xtriage (Zwart et al., 2005) to identify possible twinning and to determine a suitable resolution cutoff if desired. If the number of MR search copies is not defined, it is estimated based on the Matthews coefficient.

2.1.2. Molecular replacement. Although many ligand cocrystal structures are effectively isomorphous with the native structure and/or each other, the procedure runs Phaser (McCoy et al., 2007) by default to ensure correct placement of the protein. The search model is modified by Sculptor (Bunkóczi \& Read, 2011) to match the input sequence as closely as possible, without completing missing side chains; common modified amino acids such as phosphotyrosine are left in place if in agreement with the sequence. The default settings for the MR_AUTO mode are used, except that nonwater heteroatoms present in the search model are retained at full occupancy. If desired, the MR solution can be mapped to the same frame of reference as an isomorphous structure using phenix.find_alt_orig_sym_mate (Oeffner et al., 2012).

2.1.3. Ligand generation. If the input ligand information does not contain full geometry restraints, the molecular geometry is calculated by $e L B O W$ and output as restraints in CIF format, coordinates in PDB format and Python pickle files. Currently, the desired stereoisomer must be explicitly requested in the case of chiral ligands; although $e L B O W$ is capable of enumerating chiral centers, discrimination between enantiomers will require additional computational decisionmaking as part of the fitting procedure. Although the default optimization is usually sufficient for ligand placement, the semi-empirical AM1 quantum-mechanical method is also available and may yield improved geometries and parameters.

2.1.4. Initial refinement and rebuilding. Once the model is correctly placed, phenix.refine (Afonine et al., 2012) is run using the individual coordinate (in both real and reciprocal space) and atomic displacement parameter (ADP) refinement strategies. If Phaser was not run previously, rigid-body refinement will be performed with each protein chain as a separate group. A resolution-dependent parameterization is used for determining the ADP type and several other options, including automated rotamer fitting and solvent updating. Simulated annealing is available as an option. The user may also specify custom settings in a parameter file to be passed to phenix.refine. Weight optimization is not normally used at this stage, as rapid convergence is considered more important than obtaining an ideal geometry and minimizing overfitting.

Following the initial refinement the model is further processed to remove atoms that may interfere with ligand binding, including waters and side chains with poor fit to density. If the $R_{\text {free }}$ is greater than a specified cutoff after refinement, indicating severely misfitted regions beyond the radius of convergence of refinement, the AutoBuild wizard (Terwilliger et al., 2008) is used to apply a more aggressive strategy for improving the model (with the default rebuild-inplace mode, which will preserve the input atoms). We have found empirically that an $R_{\text {free }}$ cutoff of 0.32 is appropriate in most cases, but this can be adjusted by the user.

2.1.5. Ligand fitting. The LigandFit wizard (Terwilliger et al., 2006) is currently used for placing the target ligand (without $\mathrm{H}$ atoms) in the $m F_{\mathrm{o}}-D F_{\mathrm{c}}$ map calculated with waters removed, using the geometry specified by $e L B O W$, which produces both the restraints and coordinates in an efficient manner. The difference map may optionally be improved using an automated maximum-entropy procedure (Gull \& Daniel, 1978), which has the effect of extending it to higher resolution; however, by default maps are truncated at $1.5 \AA$, since the additional detail beyond this was found to not be beneficial (and occasionally to be detrimental) for ligand fitting owing to lower correlation coefficients even when the placement was correct. The number of ligand copies to search for is assumed to be the same as the number of copies of the search model, although this also may be specified by the user. The pipeline uses a slightly more rigorous, but slower, set of options than the settings for the default LigandFit procedure to ensure comprehensive sampling of conformations. A cutoff of 0.7 for the correlation coefficient of the ligand to the map is required for the placement to be accepted; if the results for multiple copies are inconsistent, only the highest-scoring ligands are kept. LigandFit will use NCS relationships to place ligands if possible, but still filtered by the correlation coefficient of the density fit. A post-processing step follows this with more aggressive treatment of the model, removing clashing protein atoms if a ligand copy generated from NCS operators agrees with the $2 m F_{\mathrm{o}}-D F_{\mathrm{c}}$ map. The pipeline is designed to 
easily accommodate alternative methods of ligand placement (e.g. guided ligand replacement; Klei et al., 2014).

2.1.6. Final refinement. If at least one copy of the ligand can be placed successfully, a second round of refinement is run with the following more conservative optimization strategy. If the resolution is worse than $1.75 \AA$, a grid search is used to determine the optimal weight for the X-ray and stereochemistry $/ B$-factor terms (Afonine et al., 2011). Prior to this, any amino acids with missing side-chain atoms owing to pruning or mutations made by Sculptor can optionally be automatically rebuilt and refined as an additional step. The placement of elemental ions (Echols et al., in preparation) is also offered as an option for the refinement step. Heteroatoms are sorted and grouped with the nearest chain (similar to structures deposited in the PDB).

Although the models that reach this stage are typically of high quality and near convergence, user intervention becomes unavoidable. Poorly fitted regions of the structure are usually beyond the radius of convergence of simple minimization and require manual rebuilding, and in many cases additional ligands from the buffer or crystallization conditions or missing protein residues may need to be added. As elsewhere in Phenix (Echols et al., 2012), the entire process is integrated with validation and visualization tools to streamline and encourage careful inspection of the structure. In particular, although the overall correlation coefficient is generally a reliable indicator of whether the ligand is in the correct position, the individual molecules still need to be inspected and, if necessary, corrected to verify good agreement with the electron density and prior chemical knowledge, as small local errors may be present. Following refinement, the final model is validated using the MolProbity suite (Chen et al., 2010) as implemented in Phenix and a script to view the results in Coot (Emsley et al., 2010) is generated. A simple summary file in the output directory lists each placed ligand and its fit to the electron density as judged by several metrics including overall $\mathrm{CC}$ and difference map peaks, with a warning if ligand placement was not entirely successful or if the density metrics suggest (partial) misfitting.

\subsection{Error handling}

The pipeline terminates at several logical points if relevant quality thresholds are not satisfied. If the $R_{\text {free }}$ after the initial refinement is above 0.5 , indicating an incorrect or incomplete MR solution or a model outside the normal range of convergence, no further building or ligand placement is performed. The cutoff of 0.7 for the ligand-map CC minimizes the risk of false positives, which often go undetected even with manual building (Pozharski et al., 2013). This may exclude some ligands that are in fact largely correct but include disordered fragments or are present at partial occupancy. The individual output files from LigandFit are available for manual inspection if desired. The program does not attempt to reinterpret ligand placements that pass the initial cutoff, but a postrefinement validation step calculates statistics versus the final maps and alerts the user if any values are suspicious. Our tests (data not shown) indicate that correctly placed ligands usually have a CC with the $2 m F_{\mathrm{o}}-D F_{\mathrm{c}}$ map after refinement of at least 0.9 ; values below this suggest a partial misfit and/or poor density for part of the ligand and values below 0.8 often indicate a false positive.

\subsection{Interactive mode}

To address the potential limitations of a fully automatic approach, an interactive mode is available which integrates with Coot for manual intervention. After the first refinement, Coot is opened with the refined model and maps displayed. Additional changes may then be made to the model, after which the user clicks a button to save the new model and continue the pipeline. New difference maps are calculated and passed to LigandFit. Coot is then opened a second time with a checklist for the individual ligands. Because the associated restraints CIF file is also loaded into Coot, errors in the placement can be corrected by torsion-angle rotation and real-space refinement. Ligands approved by the user are kept regardless of their initial CC from LigandFit.

\subsection{Testing}

Because our goal is to automate existing workflows, we have primarily tested structures from the PDB where the original MR search model is unambiguously annotated (either in the PDB header or in the relevant publication). In the majority of cases we reduced the model to the minimal asymmetric component, using phenix.xtriage to automatically estimate the number of copies present in the target structure based on the solvent content resulting from different numbers of copies. For ligand input, we either used the canonical SMILES string specified in the PDB (including exact chirality) or manually generated a restraints CIF file in $e L B O W$. Where necessary, the restraints needed for any additional ligands present in both the search model and published structure were generated using $e L B O W$ or phenix.ready_set. The deposited structure was used as a reference model for phenix.find_alt_orig_sym mate as described above and as an atom-name template for $e L B O W$, but the model and geometry were not otherwise used at any stage in the pipeline. For comparison, we also re-refined the published structures using the same protocol as the final refinement step of the pipeline. Ligand-atom names were adjusted as necessary to account for differences in the orientation of chemically symmetric rings (such as phenyl groups) when calculating r.m.s.d.s, without altering the chemistry or pose. All structure figures were generated with $P y M O L$ v.1.2.

\section{Results}

\subsection{Representative cases}

As examples of high-throughput applications of the pipeline, we examined several sets of related structures in detail. Most of these cases have only a single copy of a ligand and a small protein model. Run times for these examples with default settings averaged between 1 and $2 \mathrm{~h}$ on a singleprocessor core on recent AMD or Intel systems. 
Table 1

Statistics of pipeline runs for selected factor Xa (Quan et al., 2010), thrombin (Biela et al., 2012) and HIV-1 protease (Klei et al., 2007) data sets.

Unless specified by 'C' for custom, default parameters were used. For custom runs, only one attempt was made to adjust the parameters (e.g. lowering the $\mathrm{CC}$ cutoff for accepting ligand placements) and to get the refinement to complete successfully. A ' $\mathrm{P}$ ' for partial indicates that at least one of the copies of the placed ligand was mostly, but not completely, correct (e.g. it was placed at the correct location but one or more torsion angles were not set properly).

\begin{tabular}{llllll}
\hline \multirow{3}{*}{ Protein } & $\begin{array}{l}\text { PDB } \\
\text { code }\end{array}$ & $\begin{array}{l}d_{\text {min }} \\
(\AA)\end{array}$ & $\begin{array}{l}\text { Re-refined } \\
R_{\text {work }} / R_{\text {free }}\end{array}$ & $\begin{array}{l}\text { Pipeline } \\
R_{\text {work }} / R_{\text {free }}\end{array}$ & $\begin{array}{l}\text { Ligand } \\
\text { r.m.s.d. }(\AA)\end{array}$ \\
\hline \multirow{2}{*}{ Factor Xa } & 3ffg & 1.54 & $0.156 / 0.186$ & $0.159 / 0.197$ & 0.25 \\
& 3kqb & 2.25 & $0.170 / 0.193$ & $0.175 / 0.202$ & 1.21 \\
& 3kqc & 2.20 & $0.165 / 0.197$ & $0.172 / 0.209$ & 0.93 \\
Thrombin & 3kqd & 2.75 & $0.189 / 0.238$ & $0.202 / 0.239$ & 0.52 \\
& 3kqe & 2.35 & $0.173 / 0.213$ & $0.182 / 0.227$ & 0.77 \\
& 3p17 & 1.43 & $0.126 / 0.157$ & $0.140 / 0.161$ & 0.93 \\
& 3qto & 1.52 & $0.144 / 0.158$ & $0.156 / 0.172$ & 0.06 \\
& 3qtv & 1.63 & $0.144 / 0.164$ & $0.153 / 0.174$ & 0.07 \\
& 3qwc & 1.74 & $0.145 / 0.168$ & $0.152 / 0.177 \mathrm{P}$ & 3.69 \\
& & & & $0.151 / 0.173 \mathrm{C}$ & 0.08 \\
& 3qx5 & 1.35 & $0.123 / 0.146$ & $0.136 / 0.153$ & 0.06 \\
\multirow{5}{*}{ HIV-1 protease } & 3sha & 1.52 & $0.145 / 0.169$ & $0.157 / 0.178$ & 0.08 \\
& 3shc & 1.90 & $0.157 / 0.179$ & $0.162 / 0.164$ & 0.20 \\
& 3si3 & 1.55 & $0.142 / 0.164$ & $0.151 / 0.175$ & 0.17 \\
& 3si4 & 1.27 & $0.134 / 0.155$ & $0.144 / 0.163$ & 0.07 \\
& 3sv2 & 1.30 & $0.136 / 0.163$ & $0.150 / 0.172$ & 0.09 \\
& 2fxd & 1.60 & $0.181 / 0.205$ & $0.216 / 0.247$ & 0.13 \\
& 2fxe & 1.80 & $0.165 / 0.199$ & $0.179 / 0.199$ & $0.33 \dagger$ \\
\hline
\end{tabular}

$\dagger$ Single conformation only.

3.1.1. Factor Xa. The protease factor Xa has been a popular drug-discovery target, with more than 120 structures in the PDB (usually in complex with anticoagulant drug leads). We selected a set of five related phenyltriazolinone-bound structures (Quan et al., 2010), all solved at moderate resolution (2.2-2.75 $\AA$ ). Because one of these, PDB entry $3 \mathrm{ffg}$, does not have a search model defined in the PDB header, we used the search model (PDB entry 1fjs) used for entry $2 \mathrm{p} 16$ by the same depositing author (Pinto et al., 2007). The other four structures were all phased using PDB entry $3 \mathrm{ffg}$ as the search model. All of these structures were completed successfully (Table 1). The only conformational rearrangement required to accommodate the ligands was a rotation of the Asp189 side chain, which is easily accomplished by a combination of gradient minimization and rotamer fitting in phenix.refine. The automatically generated structures are very similar to the deposited models, with the exception of a flipped phenyltriazolinone moiety in PDB entry $3 \mathrm{kqb}$.

3.1.2. Thrombin. A slightly larger set of structures from an academic group is a series of compounds with human thrombin as a model system for studying the role of solvent in ligand binding (Biela et al., 2012). The structures were completed successfully by the pipeline (Table 1) using PDB entry $1 \mathrm{~h} 8 \mathrm{~d}$ as the starting model (Skordalakes et al., 2001). The final models for all but one structure have ligand conformations that are nearly identical to the published models. The exception, PDB entry $3 q w c$, differs only by a rotation of the terminal moiety, and rerunning the job using the maximumentropy procedure in map calculations resulted in a correct fit. A number of details are currently left unmodeled in the structures produced by the pipeline: $e$.g. covalently attached $\mathrm{N}$-acetylglucosamine (NAG), other small molecules such as phosphate and glycerol, misfitted side chains and alternate

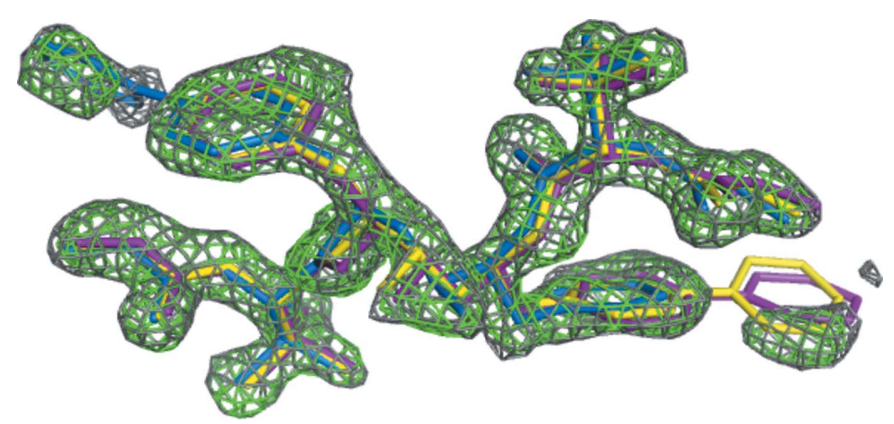

(a)

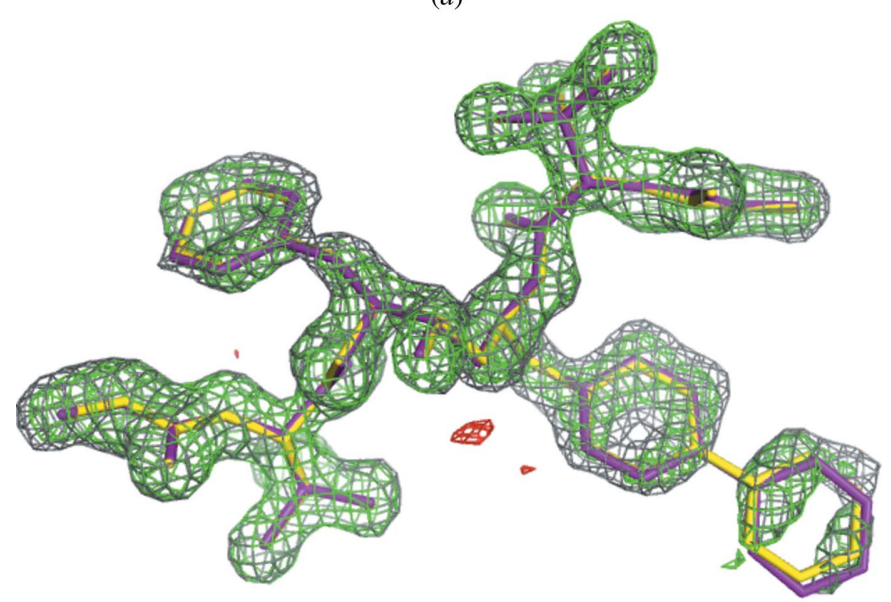

(b)

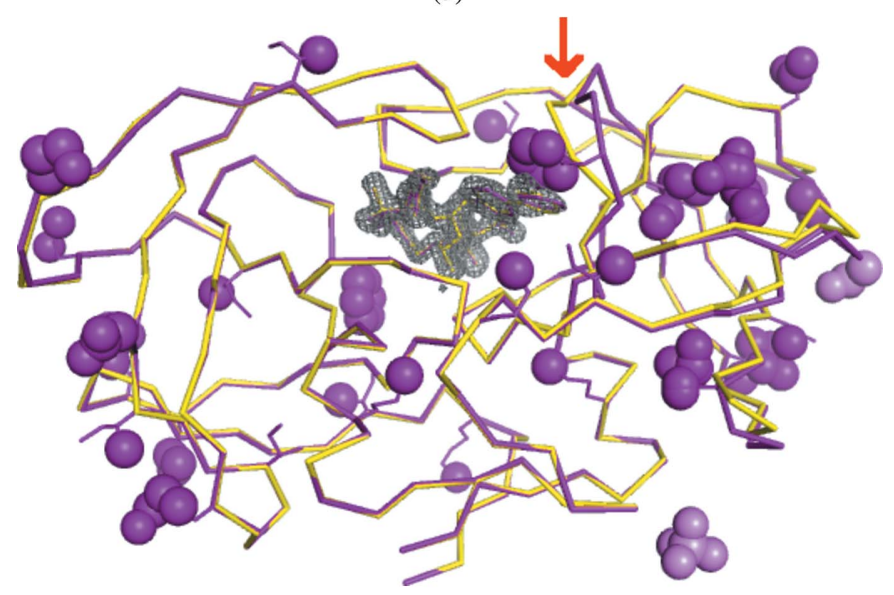

(c)

Figure 2

Comparison of refined model and ligand binding between published (purple) and pipeline (yellow) results for atazanavir-bound HIV-1 protease structures (Klei et al., 2007). The electron density prior to ligand placement is displayed as a gray mesh for the $2 m F_{\mathrm{o}}-D F_{\mathrm{c}}$ map (contoured at $1.0 \sigma$ ) and as green and red meshes for the $m F_{\mathrm{o}}-D F_{\mathrm{c}}$ map (contoured at $\pm 3.0 \sigma$ ). For clarity, only density within a $1.5 \AA$ radius of the ligand is displayed. (a) PDB entry 2fxe; active site of inhibitor-resistant mutant showing published symmetric binding of the inhibitor (with the second conformation colored blue). (b) PDB entry $2 \mathrm{fxd}$; active site of the cleavage-resistant mutant. (c) Overall structure of the cleavage-resistant mutant at the end of refinement (using chain $A$ of 2 fxe as the starting model), illustrating the remaining differences in conformation and missing atoms. The red arrow indicates the loop comprising residues $80-83$ in chain $B$. 


\section{Table 2}

Summary of the phenix.ligand_pipeline results for the Iridium test set (listed in alphabetical order by PDB code).

The re-refined $R_{\text {work }} / R_{\text {free }}$ values for the deposited models were generated using the same protocol as the final stage of the pipeline. The placed/present column gives the number of copies of the ligand placed out of the number of copies in the asymmetric unit. All copies of the target ligand were successfully placed in the first attempt for 21 of the 36 test cases; another five were successful after minor parameter adjustments. Partial solutions were obtained for six of the problematic cases (designated by italic type).

\begin{tabular}{|c|c|c|c|c|}
\hline $\begin{array}{l}\text { PDB } \\
\text { code }\end{array}$ & $\begin{array}{l}\text { Re-refined } \\
R_{\text {work }} / R_{\text {free }}\end{array}$ & $\begin{array}{l}\text { Pipeline } \\
R_{\text {work }} / R_{\text {free }}\end{array}$ & $\begin{array}{l}\text { Placed/ } \\
\text { present }\end{array}$ & $\begin{array}{l}\text { Ligand } \\
\text { r.m.s.d.(s) }(\AA)\end{array}$ \\
\hline $1 b 9 v$ & $0.175 / 0.202$ & $0.200 / 0.224$ & $1 / 1$ & 0.65 \\
\hline $1 \mathrm{br} 6$ & $0.180 / 0.224$ & $0.192 / 0.225$ & $1 / 1$ & 0.20 \\
\hline $1 c \times 2$ & $0.245 / 0.308$ & $0.250 / 0.362$ & $0 / 4$ & - \\
\hline 1exa & $0.169 / 0.192$ & $0.182 / 0.213$ & $1 / 1$ & 0.06 \\
\hline $1 \mathrm{fcx}$ & $0.135 / 0.167$ & $0.152 / 0.181$ & $1 / 1$ & 0.13 \\
\hline $1 \mathrm{fcz}$ & $0.141 / 0.175$ & $0.157 / 0.182$ & $1 / 1$ & 0.08 \\
\hline \multirow[t]{2}{*}{$1 f j s$} & $0.158 / 0.204$ & $0.231 / 0.255$ & $0 / 1$ & - \\
\hline & & $0.178 / 0.208$ & $1 / 1 C$ & 0.59 \\
\hline \multirow[t]{2}{*}{$1 \mathrm{~g} 9 \mathrm{v}$} & $0.141 / 0.166$ & $0.135 / 0.171$ & $1 / 2$ & 0.17 \\
\hline & & $0.136 / 0.171$ & $2 / 2 \mathrm{C}$ & $0.16,0.17$ \\
\hline $1 \mathrm{hpO}$ & $0.163 / 0.216$ & $0.173 / 0.227$ & $2 / 2 P$ & $0.31,2.04$ \\
\hline 1hq2 & $0.121 / 0.160$ & $0.134 / 0.166$ & $1 / 1$ & 0.10 \\
\hline 1hwi & $0.165 / 0.189$ & $0.177 / 0.198$ & $4 / 4$ & $0.32-0.44$ \\
\hline $1 h w w$ & $0.136 / 0.167$ & $0.222 / 0.248$ & $0 / 1$ & - \\
\hline $1 \mathrm{k} 3 \mathrm{u}$ & $0.135 / 0.162$ & $0.137 / 0.165$ & $1 / 1$ & 0.05 \\
\hline $112 \mathrm{~s}$ & $0.147 / 0.168$ & $0.168 / 0.195$ & $2 / 3$ & $0.15,0.16$ \\
\hline $1 \mathrm{ml} 1$ & $0.159 / 0.208$ & $0.173 / 0.208$ & $6 / 6$ & $0.25-0.86$ \\
\hline \multirow[t]{2}{*}{$1 m q 6$} & $0.171 / 0.221$ & $0.184 / 0.227$ & $1 / 1 P$ & 1.36 \\
\hline & & $0.179 / 0.230$ & $1 / 1 C$ & 0.68 \\
\hline $1 m z c$ & $0.146 / 0.168$ & $0.152 / 0.168$ & $1 / 1 P$ & 2.51 \\
\hline $1 \mathrm{n} 2 \mathrm{j}$ & $0.168 / 0.193$ & $0.185 / 0.210$ & $2 / 2$ & $0.10,0.25$ \\
\hline 1of1 & $0.156 / 0.179$ & $0.175 / 0.197$ & $2 / 2$ & $0.11,0.13$ \\
\hline 1of6 & $0.173 / 0.191$ & $0.208 / 0.230$ & $8 / 8$ & $0.11-1.82$ \\
\hline \multirow[t]{2}{*}{1 oq 5} & $0.120 / 0.164$ & $0.231 / 0.264$ & $0 / 1$ & - \\
\hline & & $0.148 / 0.188$ & $1 / 1 \mathrm{C}$ & 0.86 \\
\hline $1 \mathrm{pmn}$ & $0.190 / 0.224$ & $0.218 / 0.249$ & $1 / 1$ & 0.53 \\
\hline 1q1g & $0.156 / 0.185$ & $0.195 / 0.214$ & $6 / 6$ & $0.18-0.63$ \\
\hline $1 \mathrm{q} 41$ & $0.182 / 0.195$ & $0.207 / 0.222$ & $2 / 2$ & $0.19,0.30$ \\
\hline 1qhi & $0.214 / 0.253$ & $0.348 / 0.403$ & $0 / 1$ & - \\
\hline $1 \mathrm{r} 9 \mathrm{o}$ & $0.162 / 0.193$ & $0.240 / 0.284$ & $1 / 1$ & 0.39 \\
\hline $1 \mathrm{tt} 1$ & $0.147 / 0.171$ & $0.158 / 0.182$ & $2 / 2$ & $0.17,0.19$ \\
\hline $1 u 4 d$ & $0.187 / 0.206$ & $0.200 / 0.219$ & $2 / 2 P$ & $0.64,0.67$ \\
\hline \multirow[t]{2}{*}{ 1unl } & $0.190 / 0.214$ & $0.264 / 0.291$ & $0 / 1$ & - \\
\hline & & $0.246 / 0.276$ & $1 / 1 \mathrm{C}$ & 0.98 \\
\hline $1 \mathrm{w} 1 \mathrm{p}$ & $0.196 / 0.230$ & $0.225 / 0.257$ & $2 / 2$ & $0.22,0.30$ \\
\hline $1 \mathrm{w} 2 \mathrm{~g}$ & $0.177 / 0.198$ & $0.204 / 0.221$ & $2 / 2$ & $0.26,0.56$ \\
\hline 1 1yq & $0.201 / 0.246$ & $0.351 / 0.411$ & $0 / 1$ & - \\
\hline $1 \mathrm{yv} 3$ & $0.151 / 0.184$ & $0.169 / 0.193$ & $1 / 1$ & 0.19 \\
\hline \multirow[t]{2}{*}{ 2ack } & $0.159 / 0.185$ & $0.446 / 0.507$ & - & - \\
\hline & & $0.165 / 0.193$ & $1 / 1 \mathrm{C}$ & 0.42 \\
\hline $2 \mathrm{br} 1$ & $0.162 / 0.195$ & $0.170 / 0.212$ & $1 / 1$ & 0.33 \\
\hline $4 \cos$ & $0.205 / 0.30$ & $0.256 / 0.361$ & $0 / 4$ & - \\
\hline
\end{tabular}

conformations. However, a pair of sodium ions present in the published models were automatically built by phenix.refine in eight of the structures using a novel identification procedure (Echols et al., in preparation).

3.1.3. HIV-1 protease. Human immunodeficiency virus (HIV) protease was one of the earliest and most successful targets of structure-based drug discovery (Wlodawer, 2002). Because of its clinical importance and the rapid mutation of the viral genome, which often leads to drug resistance, more than 500 structures of various forms of the protein have been deposited in the PDB. Inhibitors typically bind in the cleft formed by the dimer, often in two symmetric poses. Because of this behavior and the frequency of mutations that affect binding, it poses a more challenging test case for automation. We tested the pipeline on a pair of mutant forms bound to the inhibitor atazanavir (Klei et al., 2007). One of these, designated the inhibitor-resistant mutant (PDB entry 2fxe), is similar to the wild-type structure and binds atazanavir symmetrically; this structure was phased using PDB entry $1 \mathrm{hvi}$ (Hosur et al., 1994). The refined structure was subsequently used to phase the cleavage-resistant mutant (PDB entry 2fxd), which binds the inhibitor in a single orientation and exhibits more local conformational differences relative to the wild type.

Because the SMILES string for atazanavir in the PDB (residue code DR7) does not specify the chirality of one of the $\mathrm{N}$ atoms, we generated the molecular structure manually in eLBOW. Although LigandFit was able to find both conformations of the ligand in PDB entry 2fxe (LigandFit typically finds five candidate placements of a ligand), only the one with the highest $\mathrm{CC}$ is selected (Fig. 2a). Either the protease monomer or the assembled dimer can be used for the input model, with essentially the same outcome. When run with a monomer the pipeline attempts to find two copies of the ligand, but since overlapping placements are not allowed it continues with the single copy and generates a warning at the end of the run. Aside from the lack of a second conformer in PDB entry $2 \mathrm{fxe}$, the automatic ligand placements for both structures are nearly identical to the deposited models (Figs. $2 a$ and $2 b$ ). The refined model for PDB entry $2 \mathrm{fxe}$ is in very close agreement with the published model and is nearly final aside from some missing side-chain atoms resulting from point mutations. Additional manual work is required to complete the PDB entry $2 \mathrm{fxd}$ model (Fig. 2c); in addition to some incomplete side chains several poorly ordered loops need inspection and possible deletion, in particular residues 80-83 in chain $B$. The optional side-chain completion step is able to restore many of the missing atoms, but the backbone conformation of some residues is sufficiently different to be outside the radius of convergence of the default refinement protocol. Both structures also have several additional unmodeled ligands (acetate, sulfate and glycerol) from the crystallization buffer.

\subsection{Benchmarking against a diverse test set}

As a more thorough measure of performance, we ran the pipeline on a set of manually curated structures used for testing ligand-docking software (the Iridium-HT test set; http://www.eyesopen.com/iridium), which have been filtered to contain only ligands whose chemical identity is unambiguous with good fit to electron density and no geometrical problems (Warren et al., 2012). We selected 36 structures representing 31 unique proteins (Supplementary Table S1 ${ }^{\mathbf{1}}$ ) for which a single search model can be unambiguously identified from the PDB header. In each case a single ligand species of interest is bound, although some structures also contain additional physiologically relevant ligands that are

\footnotetext{
${ }^{1}$ Supporting information has been deposited in the IUCr electronic archive (Reference: LV5055).
} 
present in the starting models (such as haem in PDB entry $1 \mathrm{~g} 9 \mathrm{v}$ or an ATP analog in PDB entry $1 \mathrm{hq} 2)$. The ligands vary widely in size and structure, from pantoate $\left(\mathrm{C}_{6} \mathrm{H}_{11} \mathrm{O}_{4}\right)$ to large drug-like molecules. The pipeline results (Table 2), when run with default settings, can be summarized as follows.

(i) $21(58 \%)$ of the structures (PDB entries $1 \mathrm{br} 6,1 \mathrm{~b} 9 \mathrm{v}, 1 \mathrm{exa}$, 1fcx, 1fcz, 1hq2, 1hwi, 1k3u, 1ml1, 1n2j, 1of1, 1of6, 1pmn, 1q1g, 1q41, 1r9o, 1tt1, 1w1p, 1w2g, 1yv3 and 2br1) worked unambiguously with default settings and without intervention; manual inspection confirmed that the ligand placement was essentially correct, with only minor disagreements with the published model (if any). For PDB entry 1of6, we used TYR (L-tyrosine) as the target residue based on visual inspection of the deposited model, which is incorrectly labeled as containing DTY (D-tyrosine).

(ii) PDB entry $1 \mathrm{~g} 9 \mathrm{v}$ ran successfully, but the number of copies of ligands was manually specified because the estimated number of copies of the ligands was incorrect (owing to the use of the complete hemoglobin tetramer as the search model versus two ligands bound).

(iii) PDB entry 112 s also ran successfully, but a third copy of the ligand bound between the two monomers was not built. Both active-site ligands were placed identically to the published structure, but the search for the third failed owing to the interference of a reoriented Gln side chain in the search model. (iv) The pipeline initially failed to solve PDB entry 2 ack owing to the number of copies of the protein being estimated incorrectly, resulting in an $R_{\text {free }}$ above the cutoff for continuing; re-running with this explicitly specified was successful without further intervention.

(v) The pipeline also failed on PDB entry 1oq5 owing to a poor CC for the ligand density, despite nearly perfect placement (Fig. 3a). Re-running with a more permissive CC cutoff of 0.6 was successful.

(vi) PDB entry 1mq6 runs to completion, but one section of the ligand was misfitted owing to ambiguous difference density (Fig. $3 b)$. A second run with maximum-entropy map treatment improved the density enough to result in successful placement (Fig. 3c).

(vii) Two structures, PDB entries 1fjs and 1unl, were both unsuccessful in the initial run of the pipeline but could be recovered using the maximum-entropy map calculation (with the exception of an omitted phenyl ring with poor density in 1unl).

(viii) Two structures, PDB entries $1 \mathrm{mzc}$ and $1 \mathrm{u} 4 \mathrm{~d}$, finished without error with one or more ligands placed at the expected site(s) but either failed to place all copies requested or had significant errors in the ligand conformation, geometry or orientation upon visual inspection. Some of these were easily remedied with minor adjustments in Coot.

(ix) PDB entry 1hww failed because the ligand (swainsonine, residue code SWA) consists of a flexible double-ring

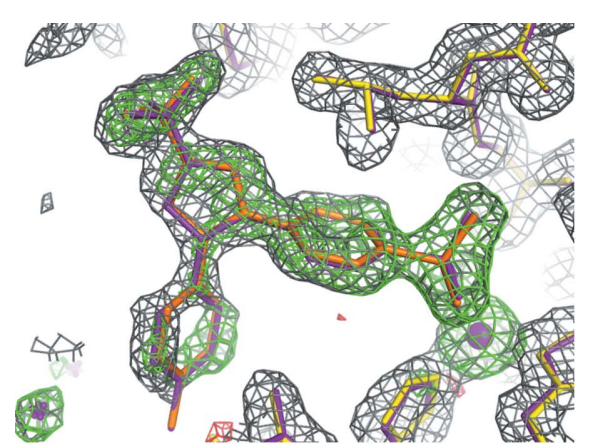

(a)

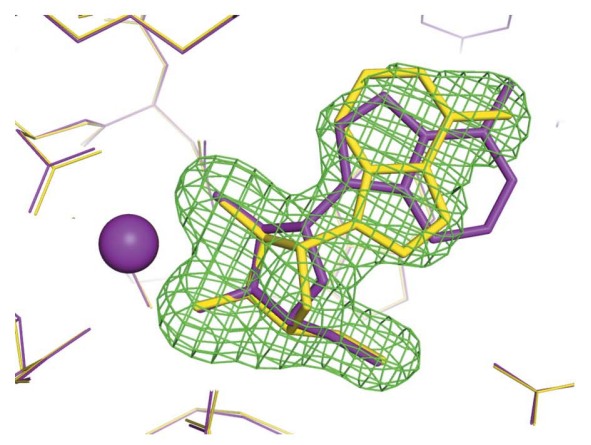

(d)

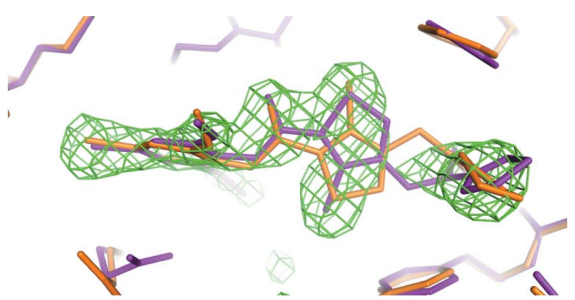

(b)

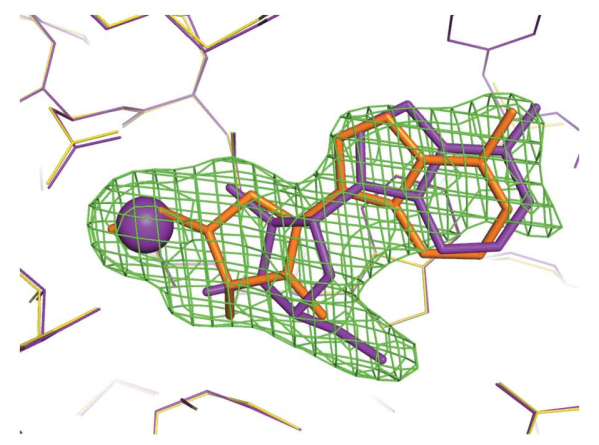

(e)

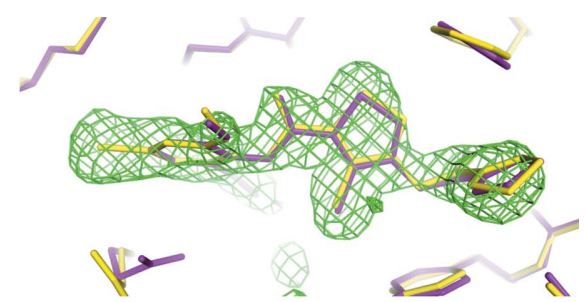

(c)

Figure 3

Examples of problematic structures in the Iridium test set. Electron density after the first round of refinement (prior to ligand fitting) is displayed as a gray mesh for the $2 m F_{\mathrm{o}}-D F_{\mathrm{c}}$ map (contoured at $1.0 \sigma$ ) and as green and red meshes for the $m F_{\mathrm{o}}-D F_{\mathrm{c}}$ map (contoured at $\pm 3.0 \sigma$ ). The published model is shown as purple sticks. Yellow sticks represent the refined model at the end of pipeline execution; orange sticks represent incorrect or rejected ligand placements. (a) PDB entry 1oq5. The ligand is correctly placed but is rejected because the CC to the $m F_{\mathrm{o}}-D F_{\mathrm{c}}$ map falls below the default cutoff of 0.7 . (b) PDB entry $1 \mathrm{mq} 6$. The ligand is partially misfitted in the run with default settings (orange sticks) owing to ambiguous $m F_{\mathrm{o}}-D F_{\mathrm{c}}$ density. Filtering the map with a maximum-entropy procedure results in correct placement $(c) .(d)$ Ligand placement in $1 \mathrm{hp} 0$, showing deviation from the published structure. (e) Misfitted ligand in $1 \mathrm{hp} 0$ with calcium ion (purple sphere) removed from the starting model. 
system that needs to be nonplanar to correctly fit the density, a degree of freedom not currently explored by LigandFit.

(x) Four structures (PDB entries 1cx2, 1qhi, 1yqy and 4cox) required more extensive rebuilding of the placed search model before ligand placement can be successful and were aborted early.

In summary, these tests indicate that use of the pipeline with default parameters is likely to be successful in a high percentage of cases (more than 50\%), while the adjustment of one or more parameters may be required for optimal success in another $30 \%$ of cases. The failure rate owing to pathological problems with ligand structure or large structural differences between the search and final model is surprisingly small at $15 \%$.

As the intention of the pipeline is to solve, fit and refine protein-ligand complexes, the output models are not publication-ready and require varying degrees of intervention to replace missing or mutated side chains, rebuild loops or place additional ligands. In PDB entry 1r9o, for instance, the search model (PDB entry 1n6b) has only $76 \%$ sequence identity and although ligand placement was successful, there are large regions on the surface of the protein that have undergone significant conformational changes and require rebuilding or deletion. However, in ten cases the final $R_{\text {free }}$ for a successful run was within $1 \%$ of the re-refined deposited structure and the geometry quality was consistently high, with a MolProbity clashscore (Chen et al., 2010) in the single digits for all successfully completed runs. For many of the examples, it is likely that an alternative search model is now available that more closely resembles the crystallized conformation and would significantly improve convergence; however, we restricted our tests to using the original search models specified by the authors.

In most structures in which ligand placement is successful, the majority of the runtime is accounted for by refinement, particularly when running weight optimization (Supplementary Table S2). Running Phaser has a relatively small impact on the overall runtime, since most MR solutions are unambiguous (and in most of the test cases there was only a single component to place). LigandFit is typically the next most time-consuming step and this time scales with the number of copies of the ligand. Because both phenix.refine and LigandFit can use multiple processor cores on Linux and Macintosh systems, the elapsed runtime can be significantly shortened on multi-core systems. However, for large sets of similar structures such as those discussed in the previous section, processing multiple data sets in parallel with a single processor per job may be a significantly more efficient use of computing resources. If desired, the execution time may be reduced by disabling weight optimization or by running LigandFit in 'quick' mode, at the expense of potentially poorer output model quality and possible failure of ligand placement.

\section{Discussion}

The procedure described here has been exercised on hundreds of structures in the PDB (data not shown) with the goal of ensuring robust behavior regardless of the ultimate outcome. Owing to the conservative criteria for evaluating the LigandFit results, the number of false positives (where a ligand is placed in the wrong site) has proven to be very low when used with default parameters. In favorable cases, where the crystallized protein has minimal changes relative to the starting model, the final structure is very nearly complete and can easily be finalized by a single round of manual inspection/ correction and refinement. In several tests, the $R_{\text {free }}$ was lower than the published structures. Although this reduction is likely to be partially owing to improvements in refinement protocols and/or under-refinement of the original models (Joosten et al., 2009; Afonine et al., 2012), it does demonstrate the ability of an automated pipeline to produce relatively high quality structures. However, we also encountered situations that are challenging for automation and potentially also for manual analysis.

Even if the model is extremely accurate and complete, limitations in map quality can hamper automatic identification of the correct binding site. In some examples (such as PDB entry 1oq5 in the Iridium test set), LigandFit places the ligand(s) correctly but the pipeline rejects these models owing to a poor CC to the difference map. Alternatively, the presence of additional unmodeled blobs of difference density may be fitted preferentially, although such false positives are usually also rejected based on the CC. These limitations on sensitivity may make the pipeline less optimal for fragmentbased drug discovery, where the ligands are typically smaller and bind with lower affinity (and partial occupancy). More flexibility may be required in the ligand-fitting step for these structures, such as fitting to the $2 m F_{\mathrm{o}}-D F_{\mathrm{c}}$ map and using a more sensitive metric than the CC. However, we found the use of maximum-entropy maps to be very helpful for several of the test cases, as it effectively increases the resolution of the Fourier map and eliminates the bulk of noise (Collins, 1982). Although the pipeline can be run in a more permissive mode by decreasing the CC cutoff and/or searching for more copies of the target ligand, this is not guaranteed to place weakly defined ligands correctly, as the presence of additional unmodeled density (for protein or other buffer components) may frustrate the fitting procedure.

More generally, the use of relatively simplistic geometry restraints instead of a physically realistic force field may limit the accuracy of ligand placement in ways that are not easily detectable by automated procedures. In particular, although the refinement is performed with explicit $\mathrm{H}$ atoms, the lack of attractive forces or solvation effects may miss fine chemical detail such as hydrogen bonding and hydrophobic interactions. The use of molecular-mechanics force fields for crystallographic refinement has been shown to yield improved protein geometry in some cases (Koparde et al., 2011; Schnieders et al., 2011; Bell, Ho et al., 2012) and it may help to overcome limitations inherent to low-resolution data sets. Refinement against a quantum-mechanical potential may also produce more accurate geometry (Li et al., 2009).

In our tests, the most common reason for failure of ligand placement was the presence of large conformational differ- 
ences from the true structure, even after the first cycle of refinement. In many structures, such as protein kinases, significant conformational changes on the scale of small loops (e.g. P-loop, DFG loop, activation loop) to entire domains (e.g. the $\mathrm{N}$-terminal lobe) accompany ligand binding. These movements are usually outside the range of advanced refinement protocols, such as simulated annealing and the deformable elastic network method (Schröder et al., 2007; Brunger et al., 2012), and instead require extensive rebuilding. Although misfitted residues can be removed from the model, aggressive pruning often results in ligand placement attempting to utilize the difference density for the removed protein atoms rather than only focusing on the true binding site. Because the current approaches to automated model building in Phenix (Terwilliger et al., 2008) are aimed at either de novo building into an experimental map or minor changes to an existing model, we have not made extensive use of them in the context of the pipeline. However, targeted application of loop-fitting methods and inference from related structures may overcome the rebuilding problem without greatly increasing the runtime. It is also likely that many structures can be solved more effectively by automatically testing multiple search models in molecular replacement (Keegan \& Winn, 2007; Long et al., 2008; Bunkóczi et al., 2013). The large numbers of PDB entries closely related to most popular drug targets offer an additional source of structural diversity that could be utilized in rebuilding.

In challenging cases, use of the interactive mode can efficiently help address problems. Such cases are readily identified after a first round of automated use of the pipeline. Additionally, for a series of related compounds, once the structure of the first protein-ligand complex has been solved, it can be used as the starting model for the remainder in an automated manner. Such was the case with the factor Xa structures (Quan et al., 2010) presented earlier. Irrespective, manual inspection of the pipeline results (a step that is streamlined by the generation of a Coot script after refinement) is required to determine the next steps for structure completion. A final round of careful validation, remediation of outstanding model deficiencies and refinement is essential before publication or deposition. Generally, further improvements in structure completion (e.g. local model rebuilding, modeling of alternate conformations, placement of small ions and additional ligands) are needed to enable researchers to generate deposition-ready structures in a fully automated manner. The adoption and diligent use of robust validation tools (Chen et al., 2010; Read et al., 2011; Pozharski et al., 2013) both during and after the structure-determination process will continue to be vital as these approaches become more sophisticated and widespread.

\section{Availability}

The program phenix.ligand_pipeline is distributed with source code in the Phenix software suite (http://www. phenix-online.org) version 1.8.3 or later. The complete suite is freely available to academic users.
We thank our colleagues in the Phenix Industrial Consortium for helpful discussions and the reviewers for their thoughts on automation and suggestions on presentation. We are grateful to Gregory Warren and OpenEye Scientific Software for curating the Iridium test set. This work was supported by the NIH (Project 1P01 GM063210) and the Phenix Industrial Consortium. This work was supported in part by the US Department of Energy under Contract No. DE-AC02-05CH11231.

\section{References}

Adams, P. D. et al. (2010). Acta Cryst. D66, 213-221.

Adler, M., Davey, D. D., Phillips, G. B., Kim, S.-H., Jancarik, J., Rumennik, G., Light, D. R. \& Whitlow, M. (2000). Biochemistry, 39, 12534-12542.

Adler, M., Kochanny, M. J., Ye, B., Rumennik, G., Light, D. R., Biancalana, S. \& Whitlow, M. (2002). Biochemistry, 41, 1551415523.

Afonine, P. V., Echols, N., Grosse-Kunstleve, R. W., Moriarty, N. W. \& Adams, P. D. (2011). Comput. Crystallogr. Newsl. 2, 99-103.

Afonine, P. V., Grosse-Kunstleve, R. W., Echols, N., Headd, J. J., Moriarty, N. W., Mustyakimov, M., Terwilliger, T. C., Urzhumtsev, A., Zwart, P. H. \& Adams, P. D. (2012). Acta Cryst. D68, 352-367.

Allingham, J. S., Smith, R. \& Rayment, I. (2005). Nature Struct. Mol. Biol. 12, 378-379.

Bell, J. A., Cao, Y., Gunn, J. R., Day, T., Gallicchio, E., Zhou, Z., Levy, R. M. \& Farid, R. (2012). International Tables for Crystallography, Vol. $F$, 2nd ed., edited by E. Arnold, D. M. Himmel \& M. G. Rossmann, pp. 534-538. New York: Wiley.

Bell, J. A., Ho, K. L. \& Farid, R. (2012). Acta Cryst. D68, 935-952.

Bennett, M. S., Wien, F., Champness, J. N., Batuwangala, T., Rutherford, T., Summers, W. C., Sun, H., Wright, G. \& Sanderson, M. R. (1999). FEBS Lett. 443, 121-125.

Berman, H., Henrick, K. \& Nakamura, H. (2003). Nature Struct. Biol. 10, 980.

Bertrand, J. A., Thieffine, S., Vulpetti, A., Cristiani, C., Valsasina, B., Knapp, S., Kalisz, H. M. \& Flocco, M. (2003). J. Mol. Biol. 333, 393-407.

Biela, A., Khayat, M., Tan, H., Kong, J., Heine, A., Hangauer, D. \& Klebe, G. (2012). J. Mol. Biol. 418, 350-366.

Blaszczyk, J., Li, Y., Shi, G., Yan, H. \& Ji, X. (2003). Biochemistry, 42, 1573-1580.

Bricogne, G., Vonrhein, C., Flensburg, C., Schiltz, M. \& Paciorek, W. (2003). Acta Cryst. D59, 2023-2030.

Broennimann, C., Eikenberry, E. F., Henrich, B., Horisberger, R., Huelsen, G., Pohl, E., Schmitt, B., Schulze-Briese, C., Suzuki, M., Tomizaki, T., Toyokawa, H. \& Wagner, A. (2006). J. Synchrotron Rad. 13, 120-130.

Brunger, A. T., Das, D., Deacon, A. M., Grant, J., Terwilliger, T. C., Read, R. J., Adams, P. D., Levitt, M. \& Schröder, G. F. (2012). Acta Cryst. D68, 391-403.

Bunkóczi, G., Echols, N., McCoy, A. J., Oeffner, R. D., Adams, P. D. \& Read, R. J. (2013). Acta Cryst. D69, 2276-2286.

Bunkóczi, G. \& Read, R. J. (2011). Acta Cryst. D67, 303-312.

Carwardine, J., Decker, G. \& Hettel, R. (2003). History of Synchrotron Radiation Sources. US Particle Accelerator School, Santa Barbara, California, June 16-20, 2003.

Chen, V. B., Arendall, W. B., Headd, J. J., Keedy, D. A., Immormino, R. M., Kapral, G. J., Murray, L. W., Richardson, J. S. \& Richardson, D. C. (2010). Acta Cryst. D66, 12-21.

Cipriani, F. et al. (2006). Acta Cryst. D62, 1251-1259.

Collins, D. M. (1982). Nature (London), 298, 49-51.

Cowtan, K. (2006). Acta Cryst. D62, 1002-1011.

Davies, T. G. \& Tickle, I. J. (2012). Top. Curr. Chem. 317, 33-59.

deSolms, S. J. et al. (2003). J. Med. Chem. 46, 2973-2984. 
DiMaio, F., Shavlik, J. \& Phillips, G. N. (2006). Bioinformatics, 22, e81-e89.

Echols, N., Grosse-Kunstleve, R. W., Afonine, P. V., Bunkóczi, G., Chen, V. B., Headd, J. J., McCoy, A. J., Moriarty, N. W., Read, R. J., Richardson, D. C., Richardson, J. S., Terwilliger, T. C. \& Adams, P. D. (2012). J. Appl. Cryst. 45, 581-586.

Elsen, J. M. van den, Kuntz, D. A. \& Rose, D. R. (2001). EMBO J. 20, 3008-3017.

Emsley, P., Lohkamp, B., Scott, W. G. \& Cowtan, K. (2010). Acta Cryst. D66, 486-501.

Finley, J. B., Atigadda, V. R., Duarte, F., Zhao, J. J., Brouillette, W. J., Air, G. M. \& Luo, M. (2000). J. Mol. Biol. 293, 1107-1119.

Fioravanti, E., Adam, V., Munier-Lehmann, H. \& Bourgeois, D. (2005). Biochemistry, 44, 130-137.

Foloppe, N., Fisher, L. M., Howes, R., Kierstan, P., Potter, A., Robertson, A. G. \& Surgenor, A. E. (2005). J. Med. Chem. 48, 4332-4345.

Grochulski, P., Fodje, M., Labiuk, S., Gorin, J., Janzen, K. \& Berg, R. (2012). J. Struct. Funct. Genomics, 13, 49-55.

Gull, S. F. \& Daniel, G. J. (1978). Nature (London), 272, 686-691.

Henrick, K. et al. (2008). Nucleic Acids Res. 36, D426-D433.

Holton, J. \& Alber, T. (2004). Proc. Natl Acad. Sci. USA, 101, 15371542

Houston, D. R., Synstad, B., Eijsink, V. G., Stark, M. J., Eggleston, I. M. \& van Aalten, D. M. F. (2004). J. Med. Chem. 47, 57135720.

Hosur, M. V., Bhat, T. N., Kempf, D., Baldwin, E. T., Liu, B., Gulnik, S., Wideburg, N. E., Norbeck, D. W., Appelt, K. \& Erickson, J. W. (1994). J. Am. Chem. Soc. 116, 847-855.

Istvan, E. S. \& Deisenhofer, J. (2001). Science, 292, 1160-1164.

Joosten, R. P., Womack, T., Vriend, G. \& Bricogne, G. (2009). Acta Cryst. D65, 176-185.

Juran, J. M. \& Gryna, F. M. (1988). Editors. Juran's Quality Control Handbook, 4th ed. New York: McGraw-Hill.

Kanda, T., Yokosuka, O. \& Omata, M. (2013). Clin. Transl. Med. $2,9$.

Karain, W. I., Bourenkov, G. P., Blume, H. \& Bartunik, H. D. (2002). Acta Cryst. D58, 1519-1522.

Keegan, R. M. \& Winn, M. D. (2007). Acta Cryst. D63, 447-457.

Klaholz, B. P., Mitschler, A., Belema, M., Zusi, C. \& Moras, D. (2000). Proc. Natl Acad. Sci. USA, 97, 6322-6327.

Klaholz, B. P., Mitschler, A. \& Moras, D. (2000). J. Mol. Biol. 302, $155-170$.

Klei, H. E., Kish, K., Lin, P.-F. M., Guo, Q., Friborg, J., Rose, R. E., Zhang, Y., Goldfarb, V., Langley, D. R., Wittekind, M. \& Sheriff, S. (2007). J. Virol. 81, 9525-9535.

Klei, H. E., Moriarty, N. W., Echols, N., Terwilliger, T. C., Baldwin, E. T., Pokross, M., Posy, S. \& Adams, P. D. (2014). Acta Cryst. D70, 134-143.

König, V., Pfeil, A., Braus, G. H. \& Schneider, T. R. (2004). J. Mol. Biol. 337, 675-690.

Koparde, V. N., Scarsdale, J. N. \& Kellogg, G. E. (2011). PLoS One, 6, e15920.

Kroemer, M., Dreyer, M. K. \& Wendt, K. U. (2004). Acta Cryst. D60, 1679-1682.

Kurumbail, R. G., Stevens, A. M., Gierse, J. K., McDonald, J. J., Stegeman, R. A., Pak, J. Y., Gildehaus, D., Miyashiro, J. M., Penning, T. D., Seibert, K., Isakson, P. C. \& Stallings, W. C. (1996). Nature (London), 384, 644-648.

Li, X., He, X., Wang, B. \& Merz, K. M. Jr (2009). J. Am. Chem. Soc. 131, 7742-7754.

Long, F., Vagin, A. A., Young, P. \& Murshudov, G. N. (2008). Acta Cryst. D64, 125-132.

Lougheed, J. C., Chen, R.-H., Mak, P. \& Stout, T. J. (2004). J. Biol. Chem. 279, 44039-44045.

Mapelli, M., Massimiliano, L., Crovace, C., Seeliger, M. A., Tsai, L.-H., Meijer, L. \& Musacchio, A. (2005). J. Med. Chem. 48, 671-679.

Mayer, M. L. (2005). Neuron, 45, 539-552.
McCoy, A. J., Grosse-Kunstleve, R. W., Adams, P. D., Winn, M. D., Storoni, L. C. \& Read, R. J. (2007). J. Appl. Cryst. 40, 658-674.

Milojkovic, D. \& Apperley, J. (2008). Curr. Opin. Oncol. 20, 112-121.

Mooij, W. T. M., Hartshorn, M. J., Tickle, I. J., Sharff, A. J., Verdonk, M. L. \& Jhoti, H. (2006). ChemMedChem, 1, 827-838.

Moriarty, N. W., Grosse-Kunstleve, R. W. \& Adams, P. D. (2009). Acta Cryst. D65, 1074-1080.

Ness, S. R., de Graaff, R. A., Abrahams, J. P. \& Pannu, N. S. (2004). Structure, 12, 1753-1761.

Oeffner, R. D., Bunkóczi, G. \& Read, R. J. (2012). Comput. Crystallogr. Newsl. 3, 5-10.

Oldfield, T. J. (2001). Acta Cryst. D57, 696-705.

Panjikar, S., Parthasarathy, V., Lamzin, V. S., Weiss, M. S. \& Tucker, P. A. (2005). Acta Cryst. D61, 449-457.

Perrakis, A., Morris, R. \& Lamzin, V. S. (1999). Nature Struct. Biol. 6, 458-463.

Pinto, D. J. P., Orwat, M. J., Koch, S., Rossi, K. A., Alexander, R. S., Smallwood, A., Wong, P. C., Rendina, A. R., Luettgen, J. M., Knabb, R. M., He, K., Xin, B., Wexler, R. R. \& Lam, P. Y. S. (2007). J. Med. Chem. 50, 5339-5356.

Powers, R. A., Morandi, F. \& Shoichet, B. K. (2002). Structure, 10, 1013-1023.

Pozharski, E., Weichenberger, C. X. \& Rupp, B. (2013). Acta Cryst. D69, 150-167.

Quan, M. L., Pinto, D. J. P., Rossi, K. A., Sheriff, S., Alexander, R. S., Amparo, E., Kish, K., Knabb, R. M., Luettgen, J. M., Morin, P., Smallwood, A., Woerner, F. J. \& Wexler, R. R. (2010). Bioorg. Med. Chem. Lett. 20, 1373-1377.

Ravelli, R. B. G., Raves, M. L., Ren, Z., Bourgeois, D., Roth, M., Kroon, J., Silman, I. \& Sussman, J. L. (1998). Acta Cryst. D54, 13591366.

Read, R. J. et al. (2011). Structure, 19, 1395-1412.

Safo, M. K., Moure, C. M., Burnett, J. C., Joshi, G. S. \& Abraham, D. J. (2001). Protein Sci. 10, 951-957.

Scapin, G., Patel, S. B., Lisnock, J., Becker, J. W. \& LoGrasso, P. V. (2003). Chem. Biol. 10, 705-712.

Schelling, P., Claus, M. T., Johner, R., Marquez, V. E., Schulz, G. E. \& Scapozza, L. (2004). J. Biol. Chem. 279, 32832-32838.

Schnieders, M. J., Fenn, T. D. \& Pande, V. S. (2011). J. Chem. Theory Comput. 7, 1141-1156.

Schröder, G. F., Brunger, A. T. \& Levitt, M. (2007). Structure, 15, 1630-1641.

Sharff, A., Flensburg, C., Keller, P., Vonrhein, C., Paciorek, W., Smart, O., Womack, T. \& Bricogne, G. (2012). Abstr. Annu. Meet. Am. Crystallogr. Assoc. 13, 22.

Shi, W., Ting, L. M., Kicska, G. A., Lewandowicz, A., Tyler, P. C., Evans, G. B., Furneaux, R. H., Kim, K., Almo, S. C. \& Schramm, V. L. (2004). J. Biol. Chem. 279, 18103-18106.

Shoop, W. L. et al. (2005). Proc. Natl Acad. Sci. USA, 102, 79587963.

Skordalakes, E., Dodson, G. G., Green, D. S., Goodwin, C. A., Scully, M. F., Hudson, H. R., Kakkar, V. V. \& Deadman, J. J. (2001). J. Mol. Biol. 311, 549-555.

Terwilliger, T. C., Adams, P. D., Read, R. J., McCoy, A. J., Moriarty, N. W., Grosse-Kunstleve, R. W., Afonine, P. V., Zwart, P. H. \& Hung, L.-W. (2009). Acta Cryst. D65, 582-601.

Terwilliger, T. C., Grosse-Kunstleve, R. W., Afonine, P. V., Moriarty, N. W., Zwart, P. H., Hung, L.-W., Read, R. J. \& Adams, P. D. (2008). Acta Cryst. D64, 61-69.

Terwilliger, T. C., Klei, H., Adams, P. D., Moriarty, N. W. \& Cohn, J. D. (2006). Acta Cryst. D62, 915-922.

Thanki, N., Zeelen, J. P., Mathieu, M., Jaenicke, R., Abagyan, R. A., Wierenga, R. K. \& Schliebs, W. (1997). Protein Eng. 10, 159167

Tsai, Y., McPhillips, S. E., González, A., McPhillips, T. M., Zinn, D., Cohen, A. E., Feese, M. D., Bushnell, D., Tiefenbrunn, T., Stout, C. D., Ludaescher, B., Hedman, B., Hodgson, K. O. \& Soltis, S. M. (2013). Acta Cryst. D69, 796-803. 
Ueno, G., Kanda, H., Hirose, R., Ida, K., Kumasaka, T. \& Yamamoto, M. (2006). J. Struct. Funct. Genomics, 7, 15-22.

Versées, W., Decanniere, K., Pellé, R., Depoorter, J., Brosens, E., Parkin, D. W. \& Steyaert, J. (2001). J. Mol. Biol. 307, 13631379.

Vonrhein, C., Flensburg, C., Keller, P., Sharff, A., Smart, O., Paciorek, W., Womack, T. \& Bricogne, G. (2011). Acta Cryst. D67, 293-302.

Wang, S. \& Eisenberg, D. (2003). Protein Sci. 12, 1097-1108.

Warren, G. L., Do, T. D., Kelley, B. P., Nicholls, A. \& Warren, S. D. (2012). Drug Discov. Today, 17, 1270-1281.

Wasserman, S. R., Koss, J. W., Sojitra, S. T., Morisco, L. L. \& Burley, S. K. (2012). Trends Pharmacol. Sci. 33, 261-267.

Weber, A., Casini, A., Heine, A., Kuhn, D., Supuran, C. T., Scozzafava, A. \& Klebe, G. (2004). J. Med. Chem. 47, 550-557.

Weininger, D. (1988). J. Chem. Inf. Comput. Sci. 28, 31-36.
Wester, M. R., Yano, J. K., Schoch, G. A., Yang, C., Griffin, K. J., Stout, C. D. \& Johnson, E. F. (2004). J. Biol. Chem. 279, 3563035637.

Weyand, M., Schlichting, I., Marabotti, A. \& Mozzarelli, A. (2002). J. Biol. Chem. 277, 10647-10652.

Winter, G. (2010). J. Appl. Cryst. 43, 186-190.

Wlodawer, A. (2002). Annu. Rev. Med. 53, 595-614.

Wlodek, S., Skillman, A. G. \& Nicholls, A. (2006). Acta Cryst. D62, 741-749.

Yan, X., Hollis, T., Svinth, M., Day, P., Monzingo, A. F., Milne, G. W. \& Robertus, J. D. (1997). J. Mol. Biol. 266, 1043-1049.

Zwart, P., Grosse-Kunstleve, R. W. \& Adams, P. D. (2005). CCP4 Newsl. Protein Crystallogr. 42, contribution 10.

Zwart, P. H., Langer, G. G. \& Lamzin, V. S. (2004). Acta Cryst. D60, 2230-2239. 